\title{
DISTRIBUCIÓN GEOGRÁFICA DEL LAGARTO GIGANTE DE EL HIERRO (Gallotia simonyi) EN EL CUATERNARIO: APLICACIÓN DE LOS DATOS PALEONTOLÓGICOS PARA LA CONSERVACIÓN DE LAS ESPECIES
}

\author{
Carolina CASTILLO', Juan J. COELLO' y \\ Esther MARTÍN GONZÁLEZ'
Dpto. de Biología Animal. Facultad de Biología. Universidad de La Laguna. 38206 La Laguna (Tenerife).e-mail: ccruiz@ull.es Laguna. 38206 La Laguna (Tenerife) \\ 2 Dpto. de Edafología y Geología. Facultad de Biología. Universidad de La
}

\begin{abstract}
Castillo, C., Coello, J.J. y Martín González, E. 2001. Distribución geográficá del lagarto gigante de El Hierro (Gallotia simonyi) en el Cuaternario: aplicación de los datos paleontológicos para la conservación de las especies. [Quaternary geographical distribution of giant lizard of El Hierro (Gallotia simonyi): Application of paleontological data for the survival of the present day species.] Revista Española de Paleontología, n. ${ }^{\circ}$ extraordinario, 15-20. ISSN 0213-6937.
\end{abstract}

\begin{abstract}
Gallotia simonyi, a lizard in danger to become extinct, is being successfully recovered in the Centro de Recuperación of Guinea (Frontera, El Hierro). The search for suitable sites for its re-introduction led to the study of its distribution in the past. The discovery of 19 paleontological sites yielding bones of $G$. simonyi evidences that its former distribution was related to the xeric and thermophyllic ecosystems existing all around the island. Even though the aborigene pressure was one of the factors that perhaps led to the nearly extinction of this species, the introduction of exotic species (cats, dogs) after the European conquest also played an important role in this problem.
\end{abstract}

Keywords: Historic distribution, conservation, reintroduction, Gallotia simonyi.

\section{RESUMEN}

El lagarto gigante de El Hierro (Gallotia simonyi) es una especie en peligro de extinción, que está siendo recuperada con éxito en el Centro de Recuperación de Guinea (Frontera, El Hierro). La búsqueda de lugares idóneos para su reintroducción ha llevado a realizar este estudio sobre su distribución en el pasado. La presencia de 19 yacimientos paleontológicos con restos de G. simonyi muestra que su antigua área de distribución estaba asociada principalmente a los ecosistemas xerófilos y termófilos de la isla. Si bien uno de los factores que pudieron conducir a la casi extinción de la especie fue la presión de los aborígenes herreños, la introducción de especies alóctonas (principalmente gatos y perros) después de la conquista europea ejerció un enorme daño sobre las poblaciones de lagarto.

Palabras clave: Distribución antigua, conservación, reintroducción, Gallotia simonyi.

\section{INTRODUCCIÓN}

El objetivo de este trabajo es dar a conocer la relación entre la información que suministra el registro fósil y su posible aplicación en la conservación del medioambiente, y en particular en la conservación y recuperación de especies en peligro de extinción. Concretamente, el estudio paleogeográfico y paleoecológico de las poblaciones fósiles puede ser útil en los programas de reintroducción de estas especies. Un ejemplo práctico de la aplicación del registro fósil a este tipo de programas lo tenemos en el estudio realizado sobre el lagarto gigante de El Hierro (Gallotia simonyi Steindachner).

El lagarto gigante de El Hierro es una especie endémica en peligro de extinción, cuya única población actual sobrevive en el Valle de El Golfo al noroeste de la isla, en un saliente rocoso del Risco de Tibataje en Frontera conocido como la Fuga de Gorreta. Aunque su distribución actual sea tan restringida, se sabe que en el pasado se extendía ampliamente por la isla; de hecho, 


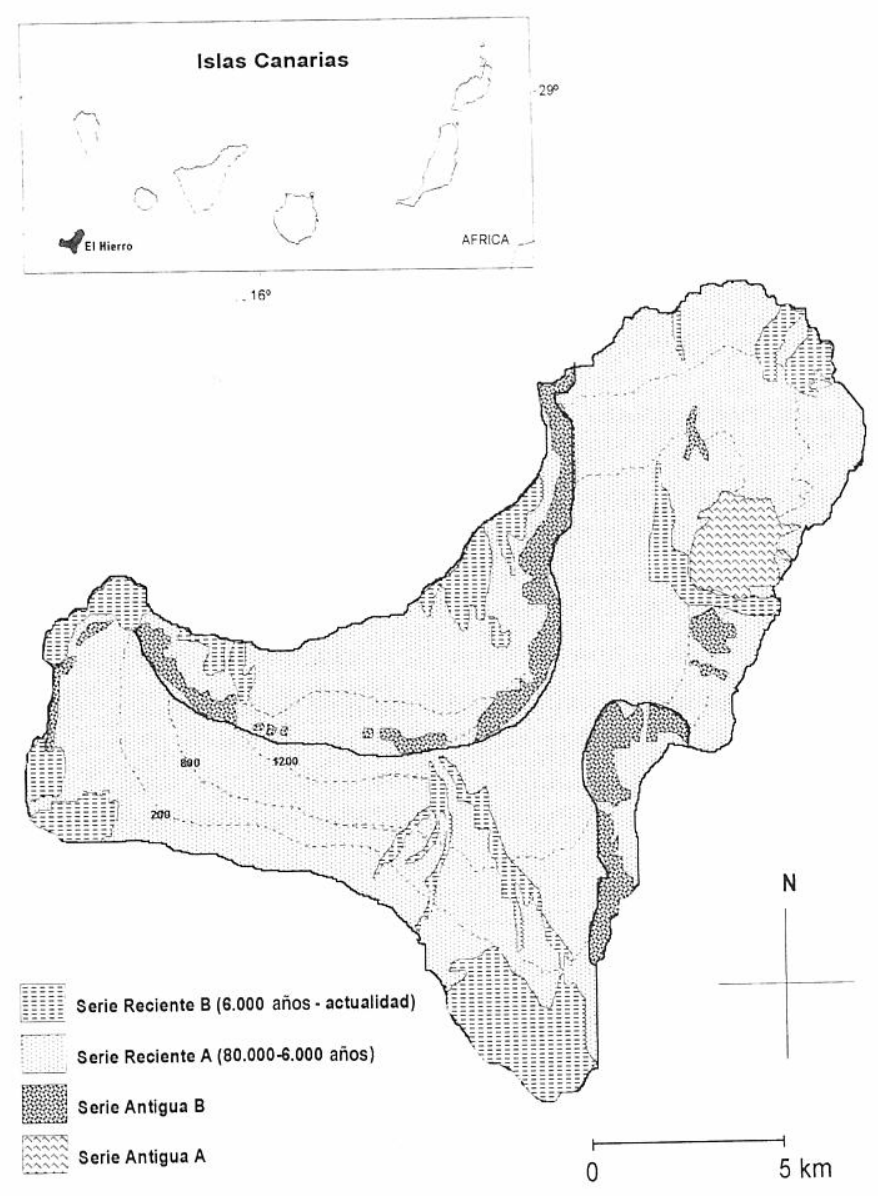

Figura 1. Mapa geológico simplificado de la isla de El Hierro (según Pellicer, 1977 y Fúster et al., 1990, modificado).

Geological map simplified of El Hierro island (sensu Pellicer, 1977 y Fúster et al., 1990, modified).

hasta hace unos 50 años existía una pequeña población en el Roque Chico de Salmor, situado frente a El Golfo, que fue extinguida como consecuencia de sobre-capturas con fines coleccionistas (Machado, 1985 a). El Plan de Recuperación de esta especie plantea como uno de sus objetivos principales la creación de nuevos núcleos poblacionales en zonas adecuadas de la isla a través de la reintroducción de individuos nacidos en cautividad en el Centro de Recuperación situado en Guinea (El Hierro) (Machado, op. cit.).

Con el fin de determinar qué lugares son los más idóneos para realizar las sueltas, es necesario, en primer lugar, conocer la distribución en el pasado de esta especie y caracterizar sus hábitats. También es necesario saber cuáles fueron los factores que llevaron a la regresión de la especie, y cuáles pueden ser las amenazas actuales, con el objeto de diseñar un programa de actuación que garantice el éxito de la reintroducción y mantenimiento de las poblaciones silvestres.

En la actualidad, en El Hierro coexisten dos especies de lagartos, el ya mencionado $G$. simonyi, y una segunda especie de menor tamaño y distribuida por toda la isla, Gallotia caesaris (Lehrs). Izquierdo et al. (1989) sugirieron la presencia en la isla de una tercera especie,
Gallotia goliath Mertens, actualmente extinta, y que se diferenciaría de las otras dos por una serie de caracteres dentarios y craneales, y por su mayor tamaño. Sin embargo, Mateo et al. (1999) sugieren que estas diferencias se explican por las variaciones ontogénicas, y que en la isla solamente han existido dos especies, coincidentes con las actuales.

\section{METODOLOGÍA}

En primer lugar se realizó una revisión bibliográfica con el objeto de recopilar información sobre todos aquellos yacimientos, tanto arqueológicos como paleontológicos, donde se hubiesen hallado restos de grandes lacértidos con anterioridad. Esta información se vio reforzada con la campaña de prospección de El Hierro con el fin de localizar nuevos yacimientos y conocer el estado de los ya existentes, visitándose un total de 39 localidades repartidas por toda la isla, y situadas en diferentes ecosistemas. Los yacimientos correspondían básicamente a dos tipos, cavidades volcánicas y formaciones sedimentarias (depósitos de ladera, depósitos de barranco y coluviones). En cada uno de los yacimientos detectados se realizó la toma sistemática de datos, en los que se incluye información geográfica, geológica y tafonómica.

Para establecer los ecosistemas en los que vivió esta especie en el pasado se comparó la distribución geográfica de los yacimientos en los que se habían encontrado restos fósiles con la distribución potencial de los pisos de vegetación actuales (Hernández Padrón et al., 1987).

La datación de los yacimientos se produjo de forma indirecta teniendo en cuenta la edad máxima y mínima de las diferentes series volcánicas existentes en El Hierro, y que la mayoría de los yacimientos estudiados se corresponden con tubos volcánicos desarrollados en esos materiales volcánicos.

El análisis tafonómico nos ha ayudado a interpretar cuáles son los factores ambientales que han actuado sobre los restos fósiles y que han conducido hacia su destrucción o conservación. Así mismo también nos ha ayudado a discernir cuáles han podido ser las causas de la cuasiextinción de G. simonyi en la isla.

\section{EL MEDIO AMBIENTE DE EL HIERRO}

El Hierro es la isla más meridional y la de menor extensión de las que forman el Archipiélago Canario. A pesar de su reducido tamaño, con un área de $278 \mathrm{~km}^{2}$, es muy escarpada y alcanza una altura máxima de 1.503 ms.n.m. en el vértice de Malpaso, situado aproximadamente en su centro geográfico.

Desde el punto de vista vulcanoestratigráfico, en los materiales volcánicos subaéreos de la isla de El Hierro se distinguen varias Series Volcánicas (Bravo, 1990; Fúster et al., 1993). En este trabajo utilizaremos una terminología propia basada en los trabajos de Bravo (op. 
cit.) y Fúster et al. (op. cit.) (Castillo y Coello, 1996) (Fig. 1). La Serie Antigua (sensu Fúster et al.,op. cit.) aflora en el sector NE de la isla, y en ella se diferencian dos series. La Serie Antigua A se formó por la actividad de la dorsal NE en el primer periodo de emisión subaérea de la isla, que comenzó aproximadamente hace $1 \mathrm{Ma}$, y terminó en 0,7 Ma. Tras este periodo de intensa actividad se produjo otro de relativa calma en el que la erosión lo desmanteló parcialmente. En la Serie Antigua B se incluyen las rocas que afloran en las paredes de El Golfo y Las Playas y en el acantilado marino del oeste.

La Serie Reciente comprende la mayor parte de la superficie de la isla, y en ella se agrupan todos los materiales que aparecen sobre la Serie Antigua en discordancias erosivas patentes, así como los conos volcánicos que tapizan toda la isla, testimonio de la gran actividad volcánica moderna. Los materiales depositados en este periodo se engloban, a su vez, en dos series recientes: A y B (Fúster et al., op. cit.). En la Serie Reciente A se incluyen todos aquellos materiales emitidos tras los deslizamientos gravitatorios de El Golfo y Las Playas (y el Julan), por lo que su límite superior de edad puede estimarse en un máximo aproximado de 80.000 años. El límite inferior de edad de esta serie se establece en unos 6.000 años, correspondiente a el último episodio eruptivo del volcán Tanganasoga (Pellicer, 1977). La Serie Reciente B (Serie Reciente de Pellicer, op. cit.) es considerada como subhistórica por Pellicer (op. cit.) y Bravo (1990) y comprende las erupciones sucedidas desde los 6.000 años hasta el presente.

La mayor parte de las simas y tubos volcánicos conocidos en la isla (Oromí et al., 1988; Hernández et al., 1992) se desarrollan en materiales superficiales pertenecientes a la Serie Reciente, cuya juventud permite que estas cavidades -formaciones geológicas muy frágiles- no hayan desaparecido por enterramiento, derrumbes internos o colmatación por sedimentos.

Los estudios florísticos y geobotánicos de la isla han permitido el establecimiento de diferentes pisos de vegetación (Hernández Padrón et al., 1987). El piso inferior, denominado Piso Basal, incluye un cinturón halófilo costero comprendido entre la línea de costa y una altura aproximada de unos $25 \mathrm{~m}$, y comunidades de plantas xerofíticas (Tabaibales y Cardonales) cuyos límites aproximados pueden establecerse entre los 5-400 $m$ de altitud (Fig. 2).

La siguiente banda altitudinal, de límites algo variables, está ocupada por comunidades vegetales xeromesófilas de transición al Piso Montano, de elevada riqueza florística. Aparecen dos comunidades principales: los sabinares húmedos, que ocupan las vertientes septentrionales de la isla, y los sabinares secos, o sabinares sensu stricto, que ocupan toda la banda sur, entre los $300 \mathrm{~m}$ y $\operatorname{los} 800 \mathrm{~m}$ de altura.

En la vertiente norte de la isla, a partir de los $600 \mathrm{~m}$ de altura, se instala una vegetación propia del Piso Montano Húmedo, o monte verde, ligada corológicamente a las precipitaciones provocadas por la formación del mar de nubes del alisio. Las elevadas pendientes del sustrato han impedido en gran parte que esta formación

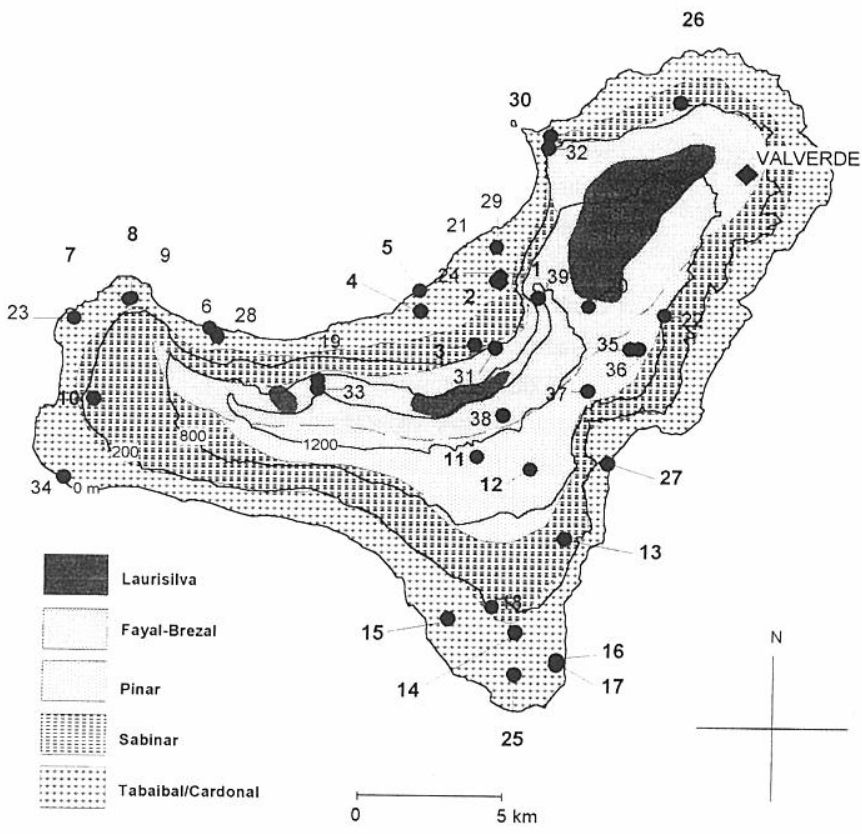

Figura 2. Mapa de vegetación donde se representan los yacimientos prospectados en la isla de El Hierro, indicándose en negrilla los que contienen restos de Gallotia simonyi. Los números se refieren a los códigos de los yacimientos que se recogen en la Tabla 1

Map of vegetation representing the localities prospected. Numbers refer to the codes stated in Table 1; those in bold correspond to sites that yielded Gallotia simonyi remains.

vegetal progrese hacia un estadio de plena laurisilva, por lo que la mayoría del monte verde herreño puede considerarse un fayal-brezal.

Por último, se encuentra la vegetación de Piso Montano Seco (Pinar), que queda limitado a la vertiente meridional de la isla, entre los $650 \mathrm{~m}$ y la línea de cumbres.

\section{RESULTADOS}

La prospección superficial de la isla de El Hierro ha dado lugar a la localización de 39 yacimientos (Fig. 2; Tabla 1), de los cuales 19 contenían restos de $G$. simonyi: 17 son cavidades (tubos y simas), y 2 son formaciones sedimentarias (coluviones) (Tabla 2). Hasta ahora el número de yacimientos de grandes lacértidos en la isla de El Hierro se elevaba a cuatro: Conchero de Playa de la Arena, Conchero de Guinea, Cuaclo de las Moleras y Cueva del Mocán. López-Jurado et al. (1999) describen 39 yacimientos más, localizados en su mayoría en la comarca de La Dehesa.

Nosotros hemos hallado nuevas localidades y hemos prospectado en algunas ya conocidas, observándose que el lagarto está repartido por toda la isla. Lo más resaltable es haber encontrado restos fósiles de $G$. simonyi en la Cueva del Curascán y en el Barranco de los Muertos en el 


\begin{tabular}{|c|c|c|c|c|}
\hline $\begin{array}{l}\text { Denominación } \\
\text { Cueva del Caserio de Guinea }\end{array}$ & $\begin{array}{c}\text { Cod. Yac. } \\
\text { H001 }\end{array}$ & $\begin{array}{c}\text { Gallotia simonyi } \\
+\end{array}$ & $\begin{array}{l}\text { Edad } \\
\text { Holoceno }\end{array}$ & $\begin{array}{c}\text { Vegetación } \\
\text { Tabaibal/Cardonal }\end{array}$ \\
\hline Cueva de Guinea & $\mathrm{H} 002$ & + & Holoceno & Tabaibal/Cardonal \\
\hline Cueva del Tío Dimas & $\mathrm{H} 003$ & + & Pleist.-Hol. & Sabinar húmedo \\
\hline Cueva Patacabra & $\mathrm{H} 004$ & + & Holoceno & Tabaibal/Cardonal \\
\hline Cueva del Submarino & $\mathrm{H} 005$ & + & Holoceno & Tabaibal/Cardonal \\
\hline Cueva del Pozo de la Salud & $\mathrm{H} 006$ & - & Holoceno & Tabaibal/Cardonal \\
\hline Cuaclo de Arenas Blancas & $\mathrm{H} 007$ & + & Holoceno & Tabaibal/Cardonal \\
\hline Sima de la Hoyeta & $\mathrm{H} 008$ & + & Holoceno & Tabaibal/Cardonal \\
\hline Sima de Narciso & $\mathrm{H} 009$ & - & Holoceno & Tabaibal/Cardonal \\
\hline Cuaclo de las Moleras & $\mathrm{H} 010$ & + & Pleist.-Hol. & Sabinar árido \\
\hline Cueva del Mocán & H011 & + & Pleist.-Hol. & Pinar \\
\hline Sima del Pico de la Mata & $\mathrm{H} 012$ & + & Pleist.-Hol. & Pinar \\
\hline Cueva de la Curva & $\mathrm{H} 013$ & + & Pleist.-Hol. & Sabinar árido \\
\hline Cueva de EI Lajial & $\mathrm{H} 014$ & + & Holoceno & Tabaibal/Cardonal \\
\hline Cueva de El Tocorón & $\mathrm{H} 015$ & + & Holoceno & Tabaibal/Cardonal \\
\hline Sima del Cráter & $\mathrm{H} 016$ & + & Holoceno & Tabaibal/Cardonal \\
\hline Cueva del Linke & $\mathrm{H} 017$ & + & Holoceno & Tabaibal/Cardonal \\
\hline Cueva Roja & $\mathrm{H} 018$ & - & Holoceno & Sabinar árido \\
\hline Cueva de Mauricio & $\mathrm{H} 019$ & - & Pleist.-Hol. & Fayal-Brezal \\
\hline Cueva de los Pozos & $\mathrm{H} 020$ & - & Pleist.-Hol. & Fayal-Brezal \\
\hline Hoyo de Isidro & $\mathrm{H} 021$ & - & Holoceno & Tabaibal/Cardonal \\
\hline Cueva del Roquillo & $\mathrm{H} 022$ & - & Pleist.-Hol. & Sabinar húmedo \\
\hline Cueva de las Pardelas & $\mathrm{H} 023$ & - & Holoceno & Tabaibal/Cardonal \\
\hline Sima de Guinea & $\mathrm{H} 024$ & - & Holoceno & Tabaibal/Cardonal \\
\hline Cueva de Don Justo & $\mathrm{H} 025$ & + & Holoceno & Tabaibal/Cardonal \\
\hline Cueva del Curascán & $\mathrm{H} 026$ & + & Pleist.-Hol. & Tabaibal/Cardonal \\
\hline Aluviones Playa de la Arena & $\mathrm{H} 027$ & + & Pleist.-Hol. & Tabaibal/Cardonal \\
\hline Coluviones Mta. Sabinosa & $\mathrm{H} 028$ & - & Pleist.-Hol. & Tabaibal/Cardonal \\
\hline Cueva de los Juaclos & $\mathrm{H} 029$ & - & Holoceno & Tabaibal/Cardonal \\
\hline Aluviones Bco. Los Muertos & $\mathrm{H} 030$ & + & Pleist.-Hol. & Tabaibal/Cardonal \\
\hline Cueva del Hoyo & $\mathrm{H} 031$ & - & Pleist.-Hol. & Fayal-Brezal \\
\hline Cueva del Risco & $\mathrm{H} 032$ & - & Pleist.-Hol. & Tabaibal/Cardonal \\
\hline Sima de las Palomas & $\mathrm{H} 033$ & - & Pleist.-Hol. & Fayal-Brezal \\
\hline Cueva del Acantilado & $\mathrm{H} 034$ & - & Holoceno & Tabaibal/Cardonal \\
\hline Cueva de la Torre 1 & $\mathrm{H} 035$ & - & Pleist.-Hol. & Pinar \\
\hline Cueva de la Torre 2 & $\mathrm{H} 036$ & - & Pleist.-Hol. & Pinar \\
\hline Cueva del Agua & $\mathrm{H} 037$ & - & Pleist.-Hol. & Pinar \\
\hline Cueva de Fileba & $\mathrm{H} 038$ & - & Pleist.-Hol. & Fayal-Brezal \\
\hline Cueva de Jinama & $\mathrm{H} 039$ & - & Pleist.-Hol. & Fayal-Brezal \\
\hline
\end{tabular}

Tabla 1. Listado de localidades prospectadas en la isla de El Hierro, indicándose los yacimientos en los que aparecieron restos de Gallotia simonyi (+), y en los que se halló otro tipo de fauna fósil (doméstica o no), o en los que no se hallaron restos (-). List of prospected localities in El Hierro island, showing the sites with Gallotia simonyi remains (+), another type of fossil fauna (domestic or not), and sites that no contain fossil remains (-).

piso bioclimático correspondiente al sabinar de la vertiente nororiental de la isla.

El rango de variación altitudinal que registran los yacimientos estudiados en este proyecto es diferente en la vertiente norte, con cotas desde 0 a $470 \mathrm{~m}$ sobre el nivel del mar, que en la sur, cuyo límite superior se establece en los $960 \mathrm{~m}$. El mayor número de yacimientos con especímenes adscritos a $G$. simonyi se localizan en el ecosistema asociado al piso basal (cardonal-tabaibal) con 14 yacimientos, seguidos por los del ecosistema de sabinar (3 yacimientos), y, por último, los del pinar (2 localidades).

La inmensa mayoría de los yacimientos se corresponden con tubos volcánicos. Los restos fósiles hallados en estas cavidades se suelen encontrar sobre el suelo de lava o englobados en el relleno sedimentario, desarticulados o semiarticulados. Las piezas pueden pertenecer a varios individuos o estar concentrados y pertenecer a un único individuo. En casi todos los casos se encuentran los restos óseos de mayor tamaño pertenecientes al esqueleto craneal o postcraneal, ya que el agua de escorrentía suele arrastrar los de menor tamaño, como falanges, vertebras, etc. Los fósiles suelen estar bien conservados, y no han experimentado una abrasión apreciable. Esto sugiere que el transporte sufrido por las piezas es muy pequeño, y que provienen de animales que murieron en el interior de las cavidades.

Cuando los restos se encuentran entre los escombros que se depositan en una sima (cavidad vertical) o bajo una grieta de gran tamaño, suelen estar muy alterados y 
frágiles. Esto podría indicar que los animales cayeron y murieron sobre los escombros, con lo que sus cadáveres quedan expuestos a factores ambientales, como pueden ser la caida de objetos, la lluvia, etc.

Seis de las localidades con restos de $G$. simonyi se corresponden con yacimientos arqueológicos y/o antropológicos ( $\mathrm{n}^{\circ} 4,7,10,11,15$, y 28) y los fósiles aparecen asociados a restos malacológicos, ictiofauna y fauna doméstica, y a veces con utensilios líticos y cerámicas. En la Cueva del Mocán (H011) muchas piezas óseas presentan un aspecto quebradizo y grisáceo que se atribuye a una cremación parcial. Además se detectó la presencia de restos de carbón y fauna doméstica entre los escombros, junto con instrumentos líticos. Sin embargo, no se ha encontrado marca alguna en los restos de lacértidos que pueda interpretarse como señal de despiece, mordisqueo o corte, y además aparecen restos de lagartos intactos -no quemados. Es posible que el fuego afectase a los restos óseos con posterioridad a su enterramiento.

\section{DISCUSIÓN}

Desde el punto de vista paleobiogeográfico, se ha puesto de manifiesto que la distribución de los grandes lagartos durante el Pleistoceno Superior y Holoceno abarca prácticamente toda la isla, incluída su porción nororiental, donde con anterioridad a este trabajo no se habían hallado sus restos (Fig. 2). La diferencia entre el rango de distribución altitudinal de $G$. simonyi en las vertientes norte y sur de la isla, parece estar asociada a la ausencia de esta especie en los ecosistemas de fayalbrezal y laurisilva actuales, y su presencia en el pinar de la vertiente meridional. A este respecto, hemos de indicar que en las 5 cavidades prospectadas en laurisilva (localidades $n^{\circ} 19,20,33,38$ y 39 de la Fig. 2) no se han encontrado restos de ningún lacértido. El papel de este piso como barrera biogeográfica en la distribución de los lagartos de las islas con este ecosistema ya ha sido apuntado (López-Jurado y Mateo, 1992). Por lo tanto, $G$. simonyi presentaba una distribución relacionada con los ecosistemas xerófilos y termófilos, desde el piso basal hasta el piso termocanario, el cual estaba dominado por sabinares en épocas pasadas, y que, en la actualidad, se halla bastante degradado, siendo ocupado por cultivos y matorrales de sustitución en muchas zonas de la isla.

La presencia del lagarto en el NE de la isla (Cueva del Curascán y Bco. de los Muertos), localizada en los dominios del sabinar húmedo y proximidades del fayal brezal, puede indicar una mayor afinidad de esta especie por los ambientes más húmedos de la isla (para afirmarlo sería necesario encontrar nuevas localidades en esta zona). Es posible que estos yacimientos se encontraran englobados en un ambiente más húmedo a principios del Holoceno, y que los cambios climáticos sucedidos en los ultimos 6.000 años (Sandweiss et al., 1999; deMenocal et al., 2000) lo hayan reducido. Estas alteraciones climáticas han conllevado cambios en la vegetación del Mediterráneo occidental y del norte de África relacionados con diferentes fases de aridificación (Jalut et al., 2000). También una posible disminución de los bosques más húmedos en la isla podría estar condicionada por las talas indiscriminadas y el aprovechamiento de la tierra después de la conquista. Sin embargo, ante la ausencia de estudios paleopolínicos que nos ofrezcan luz sobre la existencia de tales procesos en la isla de El Hierro, es necesario que los resultados obtenidos de la correlación de los yacimientos paleontológicos con los pisos bioclimáticos actuales sean tomados con precaución.

En los tubos volcánicos, los restos óseos pueden aparecer de dos formas diferentes (Castillo et al., 1996). Pueden estar depositados directamente sobre el suelo de lava, con el esqueleto articulado o disperso debido a corrientes de agua. Estos hallazgos en este tipo de yacimientos se pueden interpretar como caídas accidentales de los lagartos a través de entradas en el techo ("jameos") o grietas, y muerte por inanición, o por la entrada de los animales en estos ambientes durante épocas de menor actividad, buscando protección. Menos frecuentes son los depósitos en los que los restos se encuentran englobados en un paquete de sedimentos. Estos se han producido fundamentalmente como depósitos de escorrentía, aunque no se descarta un origen escatológico en el caso de Cueva del Linke, formado básicamente por restos de perenquenes (Tarentola sp.) y Gallotia caesaris, presas típicas de la lechuza común (Tyto alba) (Castillo et al., 2001).

Ocasionalmente los restos de lacértidos aparecen asociados a yacimientos arqueológicos, fundamentalmente "juaclos". Estos yacimientos parecen indicar que los lagartos constituían una parte de la dieta de los aborígenes, o que estos animales hacían uso de estos lugares, por ejemplo en el caso de concheros, como recurso alimenticio, sin que ambas posibilidades se excluyan (Bings, 1985; Jiménez-Gómez, 1993). Aunque de forma esporádica hemos hallado restos de grandes lagartos calcinados (yacimientos H010 y H011), este hecho puede atribuirse a causas no relacionadas directamente con su consumo. Esta presión por parte de los aborígenes pudo ser una de las causas de la disminución de las poblaciones de $G$. simonyi.

Sin embargo, las dataciones de radiocarbono (LópezJurado et al., 1999) revelan que las poblaciones de grandes lagartos eran relativamente importantes incluso después de la conquista europea. Por lo tanto la regresión de G. simonyi parece ser debida mayoritariamente a la predación por mamíferos introducidos por el hombre tras la conquista europea, destacando entre estos el gato (Machado, 1985b; López-Jurado et al., op. cit.).

\section{CONCLUSIONES}

La distribución de G. simonyi en el pasado parece haber incluido todos los pisos de vegetación de la isla (incluidos los de la vertiente nororiental), a excepción del fayal-brezal y la laurisilva, ecosistemas muy húmedos. La mayor abundancia de restos se encuentran asociados a los ambientes xerófilos, principalmente los tabaibales- 
cardonales, seguidos por los sabinares. Por ello, y a pesar del estado semidegradado de algunos de estos ambientes actualmente, a efectos de su reintroducción serían los lugares más idóneos para actuar.

Si embargo, y como uno de los principales factores que han podido conducir a la cuasi-extinción de la especie, es necesario un plan de erradicación de posibles depredadores alóctonos, como el gato o las ratas, en estas zonas.

\section{AGRADECIMIENTOS}

Los autores quieren agradecer los comentarios de los doctores Nieves Martínez López y Xabier Pereda, que ha ayudado a mejorar sustancialmente esta publicación. Este trabajo ha sido realizado a partir del proyecto Estudio de la distribución de los restos fósiles y subfósiles de Gallotia simonyi en la isla de El Hierro subvencionado por la Viceconsejería de Medio Ambiente del Gobierno de Canarias, 1996.

\section{BIBLIOGRAFÍA}

Bings, W. 1985. Zur früheren Verbreitung von Gallotia simonyi auf Hierro, mit Vorschlägen zur Wiederansiedlung. Bonner Zoologische Beitrage, 36, 417-427.

Bravo, T. 1990. Geología de la isla de El Hierro (I). Asoc. Canaria para la Enseñanza de Las Ciencias Viera y Clavijo, La Laguna, Tenerife, 9 pp.

Castillo, C. y Coello, J.J. 1996. Estudio de la distribución de los restos fósiles y subfósiles de Gallotia simonyi en la isla de El Hierro. Informe de la Viceconsejería de Medio Ambiente del Gobierno de Canarias, 77 pp.

Castillo, C., Coello, J.J., Martín Oval, M., González, C.C., Estévez, A. y Meneses, M.D. 1996. Tafonomía de las concentraciones óseas de vertebrados en los tubos volcánicos de Canarias: implicaciones paleoecológicas. In: II Reunión de Tafonomía y Fosilización (Eds. G. Meléndez Hevia, M.F. Blasco Sancho e I. Pérez Urresti). Institución Fernando El Católico (C.S.I.C.), Zaragoza, 89-96.

Castillo, C., Martín González, E. and Coello, J.J. 2001. Small vertebrate taphonomy of La Cueva del Llano, a volcanic cave on Fuerteventura (Canary Islands, Spain). Palaeoecological implications. Palaeogeography, Palaeoclimatology, Palaeoecology, 166, 277-291.

deMenocal, P., Ortiz, J., Guilderson, T. and Santhein, M. 2000. Coherent high- and low-latitude climate variability during the Holocene Warm Period. Science, 288, 21982202.

Fúster, J.M., Hernán, F., Cendrero, A., Coello, J., Cantagrel, J.M., Ancochea, E. e Ibarrola, E. 1993. Geocronología de la isla de El Hierro (Islas Canarias). Boletín de la Real Sociedad Española de Historia Natural (Sección Geológica), 88, 85-97.

Hernández, J.J., Medina, A.L. e Izquierdo, I. 1992. Volcanic caves in El Hierro Island, Canary Islands, Spain. In: 6th International Symposium on Vulcanospeleology (Ed. G.T. Rea). National Speleological Society, Alabama, USA, 185-195.

Hernández Padrón, C.E., Pérez de Paz, P.L. y Wildpret, W. 1987. Flora y vegetación liquénica epifítica de los Sabinares Herreños. Bibliotheca Lichenologica, 27. J. Cramer, Berlín, 341 pp.

Izquierdo, I., Medina, A.L. y Hernández, J.J. 1989. Bones of giant lacertids from a new site on El Hierro (Canary Islands). Amphibia-Reptilia, 10, 63-69.

Jalut, G., Esteban Amat, A., Bonet, L., Ganquelin, T. and Fontugne, M. 2000. Holocene climatic changes in the Western Mediterranean from south-east France to southeast Spain. Palaeogeography, Palaeoclimatology, Palaeoecology, 160, 255-290.

Jiménez-Gómez, M. C. 1993. El Hierro y los Bimbaches. Centro de la Cultura Popular Canaria, S/C de Tenerife, $134 \mathrm{pp}$.

López-Jurado, L. F. and Mateo, J. A. 1992. Two models of evolution in Canarian lizards based on the use of spatial resources. Biological Journal of the Linnean Society, 46, 25-37.

López-Jurado, L. F., García-Márquez, M. y Mateo, J. A. 1999. Descripción de la distribución primitiva del lagarto gigante de El Hierro y de su regresión histórica. In: El Lagarto Gigante de El Hierro. Bases para su conservación (Eds. L. F. López-Jurado y J. A. Mateo Miras). Monografías de Herpetología, 4, 17-25.

Machado, A. 1985a. Plan de Recuperación del Lagarto Gigante de El Hierro (Gallotia aff. simonyi). Ministerio de Agricultura, Pesca y Alimentación. Instituto Nacional para la Conservación de la Naturaleza, 212 pp.

Machado, A. 1985b. Conservation status of reptiles in the Canary Islands. Bonner Zoologische Beitrage, 36, 585606.

Mateo, J. A., López-Jurado, L. F. y García-Márquez, M. 1999. ¿Cuántas especies del género Gallotia había en la isla de El Hierro?. In: El Lagarto Gigante de El Hierro. Bases para su conservación (Eds. L. F. López-Jurado y J. A. Mateo Miras). Monografías de Herpetología, 4, 716.

Oromí, P., Hernández, J.J., Izquierdo, I., Martín, J.L., Medina, A.L. y Socorro, S. 1988. Catálogo de cavidades volcánicas de Canarias. I. Islas Occidentales (La Gomera, El Hierro y La Palma). G.I.E.T., Univ. La Laguna, 127 pp. (inédito).

Pellicer, M.J. 1977. Estudio volcanológico de la isla de El Hierro (Islas Canarias). Estudios Geológicos, 33, 181197.

Sandweiss, D. H., Maasch, K. A. and Anderson, D. G. 1999. Transitions in the Mid-Holocene. Science, 283, 499-500. 